\section{A descriptive online survey about the knowledge of palliative care residents of Saudi Arabia has compared to the general worldwide population}

\author{
Ibrabim Abdullah Bin Abmed, MD, SBFM, \\ Ahmed Abdullah Bin Abmed, MD, SBFM. \\ Ibrahim Abdulrahman Bin Ahmed, Medical Student, \\ Sulaiman Khalid AlFouzan, Medical Student.
}

\begin{abstract}
Objectives: To assess communities' basic knowledge of palliative care by developing a questionnaire.
\end{abstract}

Methods: This prevalence study, an anonymous online questionnaire, was answered by 326 individuals living throughout Saudi Arabia over one month. The questions concerned the basic principles and knowledge of palliative care. We collected the data between February and May 2019.

Results: The results showed that $72 \%$ of the respondents had neither heard nor knew about palliative care. Those who know about palliative care assess their knowledge as the following: $17.8 \%$ of the respondents reported that they knew the meaning and could explain it to others. As well, $10.5 \%$ knew the meaning but could not explain it to others; $9.3 \%$ had heard of it but did not know the meaning, and $62.4 \%$ had never heard of it.

Conclusion: The research showed that there is a lack of knowledge about palliative-care among the population of Saudi Arabia. Data shows that there should be more efforts toward providing the community with better knowledge about palliative care.

Keywords: palliative care, awareness, Saudi Arabia

Saudi Med J 2020; Vol. 41 (5): 537-541 doi: 10.15537/smj.2020.5.25062

$\mathrm{P}$ alliative care is the care of terminally ill patients. In Saudi Arabia, understanding of palliative care is essential to serve older adults in their local communities. The general population should have at least basic knowledge of this profession to utilize its services adequately. Palliative care is effective to the community at many levels, but might not be entirely accepted. Communities' understanding of basic, fundamental knowledge of palliative care is needed to create a well-established healthcare system. The aim of this questionnaire was to assess communities' basic, essential knowledge about palliative care. Access to palliative health is acknowledged as a human right in United Nations conventions. ${ }^{1}$

However, familiarity with palliative care varies across nations, as reported in recently published research. ${ }^{2} \mathrm{~A}$ mobile survey of a sample of 667 people in Ireland showed that many respondents were not aware of simple, basic knowledge such as terms related to end-of-life care. ${ }^{3}$ This lack of knowledge creates obstacles to ensure the quality of palliative care services provided. European researchers have hypothesized that this lack of awareness and understanding of end-of-life care among much of the public is a huge barrier to future evolution of palliative care. ${ }^{4}$ Providing appropriate care is highly significant because the world population is aging, and the incidence of chronic conditions is increasing. Consequently, many national awareness campaigns, such as dying matters by the National Council for Palliative Care in the United Kingdom, ${ }^{5}$ have been conducted to increase public knowledge and change attitudes toward dying and bereavement. ${ }^{2}$

In 2004, 2 studies were conducted in Atlantic Canada to assess the general public' level of awareness of palliative care. The first study found that $75.3 \%$ of the participants had heard about palliative care, but only $48.4 \%$ could define it appropriately. Most of the respondents who had heard about palliative care $(92.3 \%)$ agreed that they would use it if they or a family member suffered from terminal illness. In the second study, the participants read a written definition of palliative care and were then asked if they had known it before the reading. According to the results, $60.5 \%$ of the participants knew the definition before the reading, and $40.8 \%$ had first encountered palliative care during the death of a loved one. Those who did not know the definition of palliative care before the reading proposed how to increase community awareness of end-of-life care by spreading information through pamphlets, religious organizations, and media outlets among others. ${ }^{6}$

In research results, only $41 \%$ of Australians possessed the basic knowledge needed to explain the meaning of palliative care. Although $42 \%$ knew a little bit about palliative care, they did not have enough information. In addition, $17 \%$ had heard of the term palliative care but did not understand its meaning. This research found greater knowledge among women, the elderly, and office workers. The results showed that knowledge of the meaning of palliative care came from 2 primary sources: the media and personal experience. There was surprisingly little recognition of the services provided 
by palliative care, most importantly, nursing care. The study found that the participants would not research the meaning and services provided by palliative care unless they were offered the option or felt that they needed it. ${ }^{7}$

In Scotland, a survey was conducted to assess public knowledge of palliative care. Of the respondents, $49 \%$ had some information, $16 \%$ had high amounts of knowledge, and 32\% had no knowledge at all. When asked who should be offered this type of care, most strongly agreed that patients with cancer should be offered it, and only $3.7 \%(n=20)$ thought that it should not be offered to patients with HIV/AIDS. When asked "when, if they had an incurable illness, they would want information about palliative care to be provided," $64.9 \%$ of the respondents answered that they would like this information to be generally available, $20.9 \%$ at diagnosis, $12.7 \%$ when the illness became terminal, and $1.5 \%$ never. When viewing the results from the perspective of gender, the female respondents were more likely to want information to be generally accessible, while the male respondents were more likely to want information at the time of diagnosis. ${ }^{8}$

This lack of awareness to palliative care shows the need to conduct a study in Saudi Arabia to assess how much knowledge its population has and to provide solutions to increase public awareness of this magnificent work. This study was aimed at providing results to develop strategies in the future to further improve this important healthcare system, end of life care.

Methods. In this prevalence study, an anonymous online questionnaire was answered by 326 individuals living throughout Saudi Arabia over one month. The questions concerned the basic principles and knowledge of palliative care. All the questions had an option labeled "I do not know the answer" to rule out any false claims and to increase the reliability and validity of the results. The questions were direct as possible to make it easier to complete the questionnaire and to eliminate blank and false answers. Data from between February 2019 and May 2019 were collected.

Previous related articles were collected from different sources such as Journals, books, webpages, which can be found in the reference page.

Disclosure. Authors have no conflict of interests, and the work was not supported or funded by any drug company.
The study's target population was residents of Saudi Arabia because they were also the intended population to be served by palliative care services. A sample size calculator ${ }^{9}$ was used to calculate an estimated sample size of 385 with a $95 \%$ confidence interval and a 5\% margin of error. Because of limitations of distribution, 326 was the actual sample size with $92.9 \%$ confidence interval and a $5 \%$ margin of error.

For ethical reasons, only respondents 18 years or older were included. The questionnaire was posted online, and a link (https://goo.gl/forms/ eYTRmPQOGBZmptQu1) was shared on various social media platforms to reach populations with different education levels, socioeconomic status, and backgrounds. To eliminate false entries, the instructions provided with the link asked for no one living outside the country to answer it. The posted instructions also explained how to complete the questionnaire. A message with the questionnaire disclosed that the results would be used in research and that completion of the questionnaire implied consent to use the results in a study. A completion message at the end of the questionnaire indicating that the results have been submitted.

Ethical approval from the Institutional Review Board was obtained on June 2019. Only individuals 18 years or older were permitted to participate in this study for ethical reasons.

Inclusion criteria were male and females living in Saudi Arabia, above the age of 18. Exclusion criteria were: males and females less than 18 and living outside Saudi Arabia.

Depending on whether data are normally distributed or not, data will be summarized using percentages. All statistical analyses will be performed using Microsoft Excel.

Results. A total of 326 people responded to the questionnaire, and the questionnaire was carried by a survey that was limited by an online access only.

Table 1 summarize the variation of responders based on gender, socioeconomic status, and age.

The questionnaire included the following question in Arabic: "Do you know or have you ever heard about palliative care?" $72 \%$ of the respondents had neither heard nor knew about palliative care. Statistical differences in basic knowledge of palliative care appeared roughly the same between the male and female respondents: $74.4 \%$ of the female respondents and $70.7 \%$ of the male respondents had not heard of or knew about the term palliative care. Knowledge of 
Table 1 - Variation of responders based on gender, socioeconomic status, and age $(\mathrm{N}=325)$.

\begin{tabular}{lr}
\hline Variables & $\mathbf{n}(\%)$ \\
\hline Gender & \\
Male & $208(64.0)$ \\
Female & $117(36.0)$ \\
Socioeconomic status & \\
Higher than average & $111(34.3)$ \\
Average & $172(53.1)$ \\
Lower than average & $12(3.7)$ \\
Prefer not to answer & $29(9.0)$ \\
Age group (years) & \\
$<20$ & $25(7.69)$ \\
$20-39$ & $220(67.7)$ \\
$40-59$ & $62(19.1)$ \\
$\geq 60$ & $18(5.5)$ \\
\hline
\end{tabular}

In socioeconomic status one patient did complete the questionnaire

palliative care increased with the age group: $20 \%$ of 0-19 year, $27.73 \%$ of $20-39$ year, $29.0 \%$ of $40-59$ year, and $38.9 \%$ of those 60 years and older knew about or had heard of palliative care.

According to the results, $73 \%$ of higher than average socioeconomic status, $83.3 \%$ of less than average status, $72.1 \%$ of average status had not heard of or knew about palliative care. The questionnaire results showed that across socioeconomic status, the respondents had similar levels of knowledge about the basic principles of palliative care.

In response to the question "If you answered yes, what is your degree of the knowledge?", $17.8 \%$ of the respondents reported that they knew the meaning and could explain it to others. As well, $10.5 \%$ knew the meaning but could not explain it to others, $9.3 \%$ had heard of it but did not know the meaning, and $62.4 \%$ of had never heard of it. In addition, $15.4 \%$ of the female respondents and $13.46 \%$ of the male respondents knew the meaning and could explain it to others. These results showed that there were no significant gender differences in basic knowledge of palliative care.

In response to the question "If the previous question was answered yes, what is the source of your information?", $16.4 \%$ of the respondents reported that they had acquired their knowledge from work experience, $19 \%$ from personal experience, $12.1 \%$ from social media, $29.3 \%$ from family and friends, $8.6 \%$ from magazines and articles, and $31.9 \%$ from other sources. These results showed that many respondents had gained their knowledge from family and friends and personal and work experience.
For the next question "In your opinion, what do you think is the meaning of palliative care?", $80.3 \%$ of the respondents chose the correct answer. In addition, $6.5 \%$ picked "care of a patient in his home," 3.4\% "care of the elderly" $2.2 \%$ "medical care of a patient in the hospital," and $7.7 \%$ "other."

Multiple responses were allowed for the question "Which of the following is a care service provided by palliative care?". Of the respondents, $65.8 \%$ chose "pain management," $32.3 \%$ "social support for the patient and his family," 25.8\% "appropriate medical care," 25.8\% "home health care," 22.3\% "spiritual support," 24.1\% "I do not know," and $0.9 \%$ "other." According to these results, the respondents had minimal knowledge of palliative care services, such as spiritual support, home health care, and social support for patients and their families. These results showed that most respondents were not aware of the full picture of services and care provided by palliative care specialists and centers.

The next questionnaire item asked the respondents where they thought the best place to be cared for would be if they suffered from a chronic illness. This multiple-choice question included an "other" option in which the respondents could type their answers. The most common answers were "at home" (44.9\%), "at the hospital" (36\%), "at a medical care facility" (16.9\%), and "other" (2.2\%).

In response to the question "Do you know someone who is receiving palliative care or has received it in the past?", $91.1 \%$ had never encountered the specialty, while $8.9 \%$ had. This response from $91.1 \%$ of the respondents was significant because it indicated that most respondents were not sufficiently educated about the specialty to consider it as an option.

Table 2 summarized the questions carried by the survey and the frequency of the answers.

Discussion. A study conducted in Northern Ireland showed that most of the population had heard of the term palliative care but had little or no knowledge about it. ${ }^{2}$ In a study in Japan on 2 groups, the general population and those with no experience of cancer, almost two-thirds of the study population had no knowledge of palliative care, and only $0.5 \%$ had used palliative care services. ${ }^{10}$

A 2 part-study was conducted in Atlantic Canada. In the first study, the participants were asked if they had ever heard of palliative care, and those who answered yes were asked additional questions related to palliative care. Three-quarters of the study sample knew about palliative care, but only $48.4 \%$ of those who defined it correctly. In the second study, the participants read a 
Table 2 - Questions carried by the survey and the frequency of the answers.

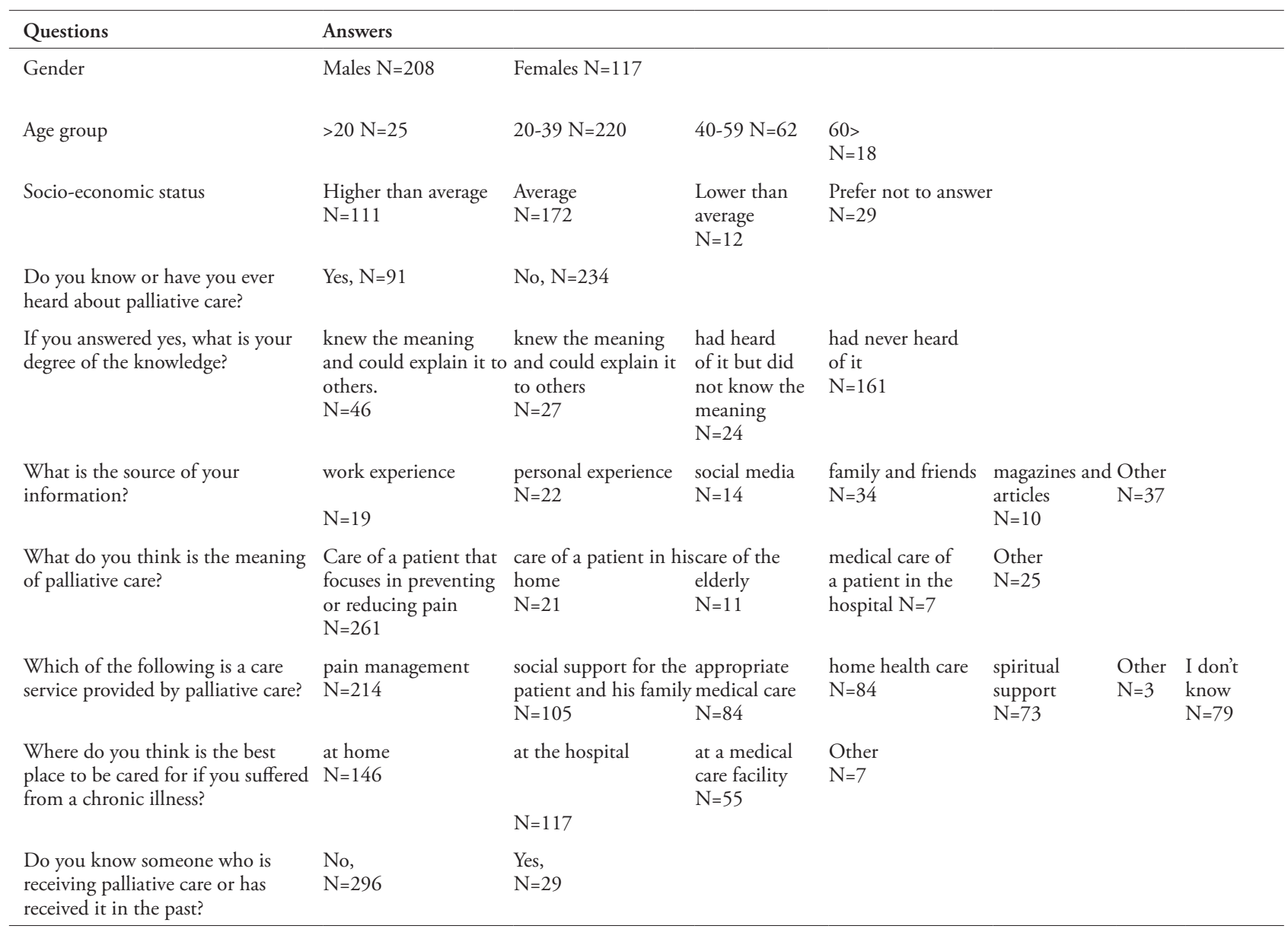

written description of palliative care and then indicated whether they had known about palliative care before reading the description. Those who answered yes were asked how they knew about palliative care. In the study results, $60.5 \%$ of the participants knew about palliative care before reading the description, and of these, $40.8 \%$ knew about it through personal experiences with family members or friends. ${ }^{6}$

This earlier research raised the question of the extent of knowledge of palliative care in Saudi Arabia. In this Saudi study, most of the respondents had neither heard of nor knew about palliative care, but most selected the right meaning, the care of the terminally ill patient, and the relief of pain. Older age groups had more knowledge, but knowledge of the basic definition of palliative care was not related to socioeconomic status, with similar results across all status levels. The questionnaire respondents acquired their knowledge from different sources, most commonly family, friends, and personal and work experience. Most selected the correct definition of palliative care, but only a minority knew all the services provided. These results showed that most respondents were not aware of the full picture of the services and care provided by palliative care specialists and centers. Moreover, $91.1 \%$ had never encountered the specialty in general. The data indicated that a significant number of the respondents were not sufficiently educated about the specialty to consider as it an option.

Most Saudi respondents had not heard of palliative care; also, they lacked the knowledge of the services provided by this specialty. In contrast to Northern Ireland, the majority heard of palliative care, but they lack the knowledge. Furthermore, the population in Canada acquire more knowledge about palliative care.

Study limitations. Future research should recruit larger, more diverse samples from more cities in Saudi Arabia. Furthermore, researchers should focus on 
strategies to improve knowledge about palliative care more and should identify barriers to palliative care and possible solutions to them. Moreover, researchers should examine palliative care services, access to them, and ways in which to improve palliative care facilities. Finally, only few of the respondents rated their experience of this specialty care, so satisfaction with the services provided in Saudi Arabia could not be determined.

Acknowledgment. We would like to thank Scribendi for English language editing. Also, we would like to thank Google forms for conducting the survey on their servers.

Received 13th October 2019. Accepted 24th March2020.

From Alfaisal University (Bin Ahmed IA), from the Department of Medicine (Bin Ahmed AA). King Abdulaziz Medical City, from the Family Medicine (Bin Ahmed IA), Imam Mohammed Bin Saud University; and from the Imam Mohammed Bin Saud University (AlFouzan), Riyadh, Kingdom of Saudi Arabia.

Address correspondence and reprints request to: Dr. Ibrahim Abdulrahman Bin Ahmed, AlFaisal University, Riyadh, Kingdom of Saudi Arabia.E-mail: I binahmed@live.com

ORCID ID: https://orcid.org/0000-0002-3051-1675

\section{References}

1. International Federation of Health and Human Rights Organization. An advocacy on palliative care as a human right. [cited 2011]. Available from: http://ifhhro.org/news-a-events/ campaign-news/275-un-advocacy-on-palliative-care-as-ahuman-right
2. McIlfatrick S, Hasson F, McLaughlin D, Johnston G, Roulston A, Rutherford L, et al. Public awareness and attitudes toward palliative care in Northern Ireland. BMC Palliat Care 2013; 12 : 34.

3. McCarthy J, Weafer J, Loughrey M. Irish views on death and dying: a national survey. J Med Ethics 2010; 36: 454-458.

4. Lynch T, Clark D, Centeno C, Rocafort J, de Lima L, Filbet M, et al. Barriers to the development of palliative care in Western Europe. Palliat Med 2010; 24: 812-819.

5. Care England. National Council for Palliative Care. Dying Matters coalition. [cited 2010]. Available from: http://www. careengland.org.uk/national-council-palliative-care-ncpc-newtoolkit

6. Claxton-Oldfield S, Claxton-Oldfield J, Rishchynski G. Understanding of the term "palliative care": a Canadian survey. Am J Hosp Palliat Care 2004; 21: 105-110.

7. Australian Department of Health and Ageing. Community attitudes to palliative care issues. Canberra (AU): Rural Health, and Palliative Care Branch, Australian Department of Health and Ageing; 2003.

8. Wallace J. Public awareness of palliative care: report of the findings of the first national survey on Scotland into public knowledge and understanding of palliative care. Edinburgh (UK): Scottish Partnership for Palliative Care; 2003.

9. Raosoft. Raosoft sample size calculator. [cited 2004]. Available from: http://www.raosoft.com/samplesize.html

10. Hirai K, Kudo T, Akiyama M, Matoba M, Shiozaki M, Yamaki T, et al. Public awareness, knowledge of availability, and readiness for cancer palliative care services: a population-based survey across four regions in Japan. J Palliat Med 2011; 14: 918-922. 